\title{
Prospección geoquímica para oro, en terrazas aluviales del rio Pastaza (C)
}

Geochemical prospection for gold, in aluvial terraces of the pastaza river

Marco Mejía. ${ }^{1} \&$ Alexis Aciptio. ${ }^{2}$

Recibido: 27-04-2019 / Revisado: 28-05-2019 /Aceptado: 28-06-2019/ Publicado: 15-07-2019

\section{Summary. DOI: https://doi.org/10.33262/cienciadigital.v3i3.1.709}

The purpose of this research was to carry out the prospecting of secondary gold in an alluvial terrace in the community of Kunkuk, by means of geochemical sampling and the application of the chemical analysis fire test.

The work was executed in four phases: first phase corresponds to the stage of document collection and sampling network design; the second involves field work, which corresponds to a direct method with the collection, recognition and study of the samples in the place where they are located. The third concerns laboratory tests, where total gold content was determined per sample collected by performing a qualitative chemical analysis.

The last phase, consists of the processing of data to obtain the results in the study area and the geo-statistical tabulation to determine the anomalous gold values in the sampling area, a delimitation of the values is also made as are Background, threshold, sub anomaly, anomaly and anomaly defined with the help of professional software expressed in graphic tables and thematic maps. With the results obtained, it was possible to demonstrate and categorize a deposit of alluvial gold and propose future more specific investigations in sectors with greater anomalous reading.

Keywords: alluvial gold - chemical analysis - geochemical prospecting

\section{Resumen:}

La presente investigación tuvo como objeto realizar la prospección de oro secundario en una terraza aluvial en la comunidad de Kunkuk, mediante muestreo geoquímico y la aplicación del análisis químico ensayo al fuego.

El trabajo se ejecutó en cuatro fases: primera fase corresponde a la etapa de recopilación documental y diseño de red de muestreo; la segunda incumbe trabajo de campo, que corresponde a un método directo con la recolección, reconocimiento y estudio de las muestras en el lugar donde están localizadas. La tercera concierne ensayos de laboratorio, donde se determinó contenido de oro total por muestra recolectada realizando un análisis químico cualitativo.

\footnotetext{
${ }^{1}$ Escuela Superior Politécnica de Chimborazo, Ecuador. mejiamarck@yahoo.com

${ }^{2}$ Escuela Superior Politécnica de Chimborazo. Ecuador. alexisaciptio@yahoo.com
} 
La última fase, consiste en el procesamiento de datos para la obtención de los resultados en el área de estudio y la tabulación geo estadística para determinar los valores anómalos de oro en el área de muestreo, también se hace una delimitación de los valores como son Background, threshold, sub anomalía, anomalía y anomalía definida con la ayuda de software profesional expresados en tablas gráficos y mapas temáticos. Con los resultados obtenidos se pudo evidenciar y categorizar un depósito de oro aluvial y plantear futuras investigaciones más puntuales en sectores de mayor lectura anomálica.

Palabras claves: oro aluvial - análisis químico - prospección geoquímica

\section{Introducción}

El Ecuador por su entorno geológico heterogéneo ha sido beneficiado con gran variedad de minerales, los cuales son requeridos actualmente y serán necesarios a futuro, a parte, el avance tecnológico permite cada día mejorar en todo ámbito lo que se traduce en un entorno esperanzador para innovar y mitigar los aspectos negativos que causa la extracción de minerales.

Un aspecto importante a destacar es la minería artesanal en el Ecuador, dedicada a la explotación de placeres de oro, la cual actualmente se realiza con poco o nada conocimiento técnico lo que repercute significativamente en los beneficios económicos por parte de quienes se dedican a esta actividad, de tal manera que envés de generar empleo y contribuir al desarrollo del país ocasiona problemas sociales, económicos y ambientales. En vista de este acontecimiento se pretende aportar de manera concreta y sencilla a una parte de la minería en el Ecuador.

El estudio a realizarse servirá como base para la realización de cualquier prospección con un alto grado de confiabilidad en diferentes tipos de placeres de oro, de tal forma que permita realizar inversiones que luego se traduzcan en réditos económicos los cuales sirvan continuamente para mejorar la explotación, mitigar el impacto y crear fuentes de trabajo

\section{Desarrollo:}

\section{Marco teórico}

Ubicación .- El área de estudio se encuentra ubicada en la provincia de Pastaza, Cantón Pastaza en la comunidad Kunkuk .

Acceso .- El acceso al área de estudio se lo realiza por medio de la vía de primer orden Macas- Puyo partiendo desde la cuidad de Macas 70 kilómetros hasta la provincia 


\section{(jiencia

de Pastaza en la comunidad Kunkuk, el área de estudio se encuentra localizada junto a la vía principal por lo que se ingresa a pie.

\section{Génesis Del Oro Aluvial En El Sector Oriental.}

En el sector oriental Distritos Chinchipe, Zamora Upano y Pastaza Napo Aguarico, que forman parte del gran Distrito Gigante de Bolivia, Perú, Ecuador y Colombia, el origen de oro aluvial es a partir de Skarns de oro tipo Nambija, vetas de cuarzo aurífero epitermales y mesotermales, zonas de cizalla mineralizadas, yacimientos diseminados tipo pórfidos de $\mathrm{Cu} \mathrm{Au}$, Stockworks y brechas.

Una reconcentración muy importante se produce a partir de Formaciones Antiguas, como la Formación Hollín que presenta oro en la parte basal, y de la Formación conglomerática Tiyuyacu que contiene horizontes auríferos. Otras formaciones como la Mera contienen también horizontes con oro aluvial. Los placeres Terciarios más importantes son de la Formación Tiyuyacu en el sector Cusuimi, Campo Canuza y Macuma, con grandes extensiones de material aurífero que puede ser aprovechado por las comunidades shuaras que habitan en el sector (Pillajo Gavidia, 2010, p. 17).

\section{Placeres Fluviales o Aluviales.}

El ambiente de depositación de este tipo de depósitos empieza en las áreas fuente, donde los sedimentos auríferos entran a las partes superiores de las corrientes, como mezclas heterogéneas de rocas, parcialmente alteradas. El oro más pesado y más grueso, se asienta más rápidamente que sus sedimentos y se puede ir hasta el fondo de la roca Basal o Peña.

El oro fino y laminar será transportado lejos de su fuente.

Esta distancia que recorre el oro fino, ha sido calculada por algunos geólogos en $40 \mathrm{~km}$; este oro no puede recuperarse con los equipos tradicionales de concentración por gravedad.

El estimado anterior da a entender que las concentraciones de oro, de diversos tamaños, que se encuentran a lo largo de un río o de un depósito aluvial, no provienen de una sola fuente, en las cabeceras de río, sino que han recibido aportes de varios afluentes secundarios en su recorrido.

Según Cadavid Mejía, p.24-25 (1998) en este tipo de depósitos las concentraciones, en las partes altas o cabeceras de las corrientes, tienen muchas variaciones con el tiempo; es decir, hay épocas de erosión y de depositación continuas. Cualquier alteración del lecho, del río por crecientes fuertes o por la mano del hombre o por otro fenómeno, arrastra lo depositado en un tiempo y lo concentra en otra parte. Los depósitos aluviales reciben diversos nombres según el tipo de concentración que hagan. 


\section{Cliencia

Entre estos mencionaremos la terraza, las cuales son de varios tipos, las concentraciones en barras (rifles) naturales de lechos de ríos; las depresiones (huecos) de los lechos; las partes superiores de las islas las partes internas de las curvas o meandros, los remolinos, la confluencia de corrientes, las concentraciones que ocurren por perdida súbita de velocidad y gradientes, tal como ocurren a la entrada y salida de un estrecho de la corriente; las concentraciones por pérdida de velocidad, debido a la carga de sedimentos en el lecho, o por la presencia de rocas y por último, las concentraciones que pueden ocurrir después de las crecientes, llamados depósitos de llanura de inundación (Cadavid Mejía, 1998, p.24-25).

\section{Prospección Geoquímica}

Consiste en el análisis de muestras de sedimentos de suelos, aguas o incluso de plantas que puedan concentrar elementos químicos relacionados con una determinada mineralización. La geoquímica del yacimiento tiene como finalidad conocer con el mayor detalle la distribución de los elementos químicos relacionados de forma directa o indirecta con la mineralización, o afectados por los procesos que han formado o modificado el yacimiento (López \& Blanco, 2010, p.24).

Los pasos de la prospección geoquímica según la (Universidad de Atacama, 2015) son los siguientes:

- Selección de los métodos de los elementos de interés, de la sensibilidad y la precisión necesarias y de la red de muestreo. Las selecciones se toman con base en los costos, los conocimientos geológicos, la capacidad del laboratorio disponible y una investigación preliminar o las experiencias con áreas parecidas.

- Programa de muestreo preliminar, que incluye análisis inmediato de algunas muestras tomadas en la superficie y en varias profundidades en el subsuelo para establecer los márgenes de confianza y para evaluar los factores, que contribuyen a su determinación.

- Análisis de las muestras en el terreno y en el laboratorio, incluido análisis por medio de varios métodos.

- Estadísticas de los resultados y evaluación geológica de los datos tomando en cuenta los datos geológicos y geofísicos.

- Confirmación de anomalías aparentes, muestreo encauzado en áreas más pequeñas (red de muestreo con espaciamiento corto), análisis de las muestras y evaluación de los resultados.

- Investigación encauzada con muestreo y análisis adicionales de muestras tomadas en un paso anterior.

\section{Muestras En Suelos Residuales}

El objetivo del estudio geoquímico de suelos consiste en el reconocimiento de la distribución primaria de elementos seleccionados en las rocas subyacentes. En los suelos 


\section{Silencia

residuales generalmente la distribución primaria se expresa todavía en forma relativamente clara, aún estará modificada por los efectos de varios procesos superficiales. Algunos de estos procesos tienden a homogeneizar el suelo y por consiguiente borrar la distribución primaria como entre otros la helada, la actividad de plantas, la gravedad, la disolución local y la redeposición. Otros procesos contribuyen a la formación de horizontes verticalmente diferenciados o es decir favorecen la formación de un suelo. Otros procesos, que tienden a borrar la distribución primaria, son la remoción de elementos mediante la meteorización y la formación del suelo (corrosión por agua meteórica, ascenso por plantas) y la adición de elementos (por deposición del agua subterránea, adición de elementos provenientes de la desintegración de vegetación, por polvos, elementos disueltos en agua meteórica).

\section{Elemento Indicador, Elemento Explorador}

Elemento indicador, indicador directo o elemento blanco ('target element') se refiere a uno de los elementos principales del depósito mineral, que se espera encontrar.

Elemento explorador o elemento pionero ('pathfinder element') se refiere a un elemento asociado con el depósito mineral, pero que puede ser detectado más fácilmente en comparación al elemento blanco, que puede ser dispersado en un área más extendida y que no está acompañado por tanto ruido de fondo en comparación al elemento blanco (Griem-Klee, 2016).

La selección de un elemento explorador requiere un modelo del depósito mineral, que se espera descubrir. Arsénico (As) por ejemplo puede presentar un elemento explorador para la búsqueda de cobre $(\mathrm{Cu})$ en un depósito macizo de sulfuros, pero no es un elemento explorador para cada tipo de depósito de cobre.

\section{Anomalías En Sedimentos De Drenaje}

A los sedimentos de drenaje pertenecen los sedimentos de manantiales, de lagos, de llanuras de inundación, los sedimentos activos de corrientes de agua y los sedimentos, que funcionan como filtros para el agua "seepage sediments".

Los sistemas de drenaje a menudo parten de manantiales. Los sedimentos situados en la cercanía de los manantiales y los sedimentos de filtración tienden a exhibir anomalías apreciables y por consiguiente estos sedimentos son útiles para una exploración geoquímica.

Los sedimentos activos de corrientes de agua incluyen material clástico y hidromórfico de los sectores de filtración, el material clástico erosionado de los bancos de material detrítico situados en los lechos de los ríos y de material hidromórfico absorbido o precipitado por el agua de escorrentía. 


\section{Cliencia

Las anomalías desarrolladas en estos sedimentos activos pueden extenderse varios kilómetros con respecto a su fuente. Los estudios de estas anomalías se utilizan frecuente y preferentemente para lograr un reconocimiento general. En el caso de los lagos se estudia los componentes clásticos y el material absorbido o precipitado de los sedimentos. En áreas con una alta cantidad de lagos como en el área del escudo precámbrico de Canadá modelado por glaciares el estudio geoquímico de los sedimentos de lagos puede ser el método más económico y efectivo para un reconocimiento general

\section{Ensayos Geoquímicos}

Consiste en medir sistemáticamente una o más propiedades químicas, principalmente el contenido de elementos menores y trazas de una sustancia o material que se presente en estado natural, como por ejemplo, rocas frescas y mineralizadas (fragmentos, núcleos de perforación y rodados), suelos, sedimentos activos fluviales o lacustres, detritos glaciales, vegetación, agua superficial y subterránea, vapor de agua, gas, aire, entre otros (Foster, 1992).

\section{Anomalía Geoquímica.}

Es una variación de la distribución geoquímica normal correspondiente a un área o a un ambiente geoquímico. Una anomalía se expresa por medio de números, que se puede separar de un grupo más amplio de números constituyendo el fondo geoquímico. Otros factores de una anomalía geoquímica de importancia son el marco topográfico y la asociación geológica (Morales, 1985, p.17).

\section{Ensayo Al Fuego}

El Método de Ensayo al Fuego consiste en producir una fusión de la muestra usando reactivos y fundentes adecuados para obtener dos fases líquidas: una escoria constituida principalmente por silicatos complejos y una fase metálica constituida por plomo, el cual colecta los metales de interés (Au y Ag); que posteriormente serán sometidos a Análisis Químico o determinación gravimétrica, según condiciones finales de la muestra.

El mineral en polvo se funde con un flux a base de plomo a $1010^{\circ} \mathrm{C}$. El oro y plata es recolectado por el régulo de plomo, luego este régulo se copela a $900{ }^{\circ} \mathrm{C}$. Medimos la masa del doré y luego se disgrega con $\mathrm{HNO} 3$ al 15\%, obteniéndose el botón de oro para que finalmente se calcine y se mida su masa. El cálculo de Oro y Plata se expresa en gr/TM (ALBEXXUS LABORATORIO METALÚRGICO Cía. Ltda, 2014

En la metodología se realizó básicamente en cuatro partes:

- Recopilación de información y elaboración de mapas preliminares 


\section{- Ciencia

- Trabajo de campo

- Análisis de laboratorio

- Trabajo de final de oficina

Cabe recalcar que en el área no existe información sobre trabajos similares por lo que toda la información necesaria para la realización del estudio será obtenida con el trabajo de campo y de laboratorio.

\section{Muestreo De Suelos}

Una vez definida el área de interés, se planificó la malla de muestreo abarcando el área de estudio. Se estableció tomar una muestra cada 150 metros a lo largo y ancho del lugar, asegurando una malla de muestreo regular lo más cerrada posible, a que en el terreno se lo puede considerar plano, Estas características morfológicas hicieron que se use una malla regular.

Como segundo paso en la recolección de muestras de suelo para el análisis de laboratorio se procedió a ubicar el punto de muestreo con ayuda de un GPS, registrando las coordenadas y dando una codificación a cada muestra.

Una vez ubicado y georreferenciado el punto de muestreo con ayuda de una pala se procede a limpiar la cobertura vegetal existente, y con ayuda del muestreador Auger se procede a sacar una muestra de suelo de $4 \mathrm{~kg}$ aproximadamente.

La muestra de grava es cuarteada y se reduce su peso hasta tener una muestra de 1 kilogramo la misma que será enviada a laboratorio para su análisis, se procede a anotar las características físicas de la muestra como coloración, humedad y profundidad a la cual ha sido tomada.

Finalmente, ya cuando la muestra ha sido tomada se procede a rellenar el sondeo exploratorio con el mismo material restante que se retiró inicialmente

\section{Marco metodológico}

Para efectuar este trabajo investigativo en las terrazas aluviales del rio Pastaza, en la comunidad de Kunkuk Provincia de Pastaza se procedió a determinar y ubicar los sitios idóneos para el muestreo de campo a lo cual acudimos a mapa geológico, carta topográfica e indicios físicos apreciados en recorridos previos, o de conocimiento tradicional proporcionados por los moradores, considerando de manera particular que la zona presenta sitios pantanosos y con características de gran carga acuífera se traza una malla regular de $150 \mathrm{~m}$ para la toma de muestra; inmediatamente se dio inicio a la fase de trabajo de campo. 


\section{Cliencia

Se utilizó el muestreo geoquímico generalmente es la forma corriente de obtener muestras representativas en sedimento aurífero "seepage sediments"., colectado forma sistemática mediante malla regular de cada 150 metros. La cantidad de material recogido como muestra de $4 \mathrm{~kg}$ mediante muestreador de suelo para entubación, el mismo que es enfundando sellado y marcado con un código

Para realizar el método de Ensayo al Fuego consiste en producir una fusión de la muestra usando reactivos y fundentes adecuados para obtener dos fases líquidas: una escoria constituida principalmente por silicatos complejos y una fase metálica constituida por plomo, el cual colecta los metales de interés ( $\mathrm{Au}$ y Ag); que posteriormente serán sometidos a Análisis Químico o determinación gravimétrica, según condiciones finales de la muestra.

El mineral en polvo se funde con un flux a base de plomo a $1010^{\circ} \mathrm{C}$. El oro y plata es recolectado por el régulo de plomo, luego este régulo se copela a $900{ }^{\circ} \mathrm{C}$. Medimos la masa del doré y luego se disgrega con $\mathrm{HNO} 3$ al 15\%, obteniéndose el botón de oro para que finalmente se calcine y se mida su masa. El cálculo de Oro y Plata se expresa en gr/TM (ALBEXXUS LABORATORIO METALÚRGICO Cía. Ltda, 2014).

Para el análisis e interpretación de los datos obtenidos en laboratorio nos basamos en el método de Lepeltier modificado, mediante el cual definimos los valores anomálicos de un elemento como son el Background es el equivalente a la media aritmética, Threshold igual a la suma de la media aritmética más la desviación estándar, la Sub-anomalía es igual a la suma de dos desviaciones estándar más la media aritmética y la Anomalía desviaciones estándar más la media aritmética, a continuación se presentan los resultados obtenidos de los cálculos estadísticos realizados.

\section{Población sobre la que se ha hecho el estudio:}

La superficie de estudio comprende una extensión 45 hectáreas en la comunidad de Kunkuk Provincia de Pastaza". De las cuales se les considero netamente 30 hectareas accesibles y con el salvo conducto correspondiente.

Entorno: El sector es netamente rural, conformado por una planicie amazónica tipo fluvial. compuesta por material detrítico, de granulación muy fina a grava, la zona está parcialmente inundada, cubierta por revegetación autóctona tipo amazónica

\section{Intervenciones:}

Se tomó 22 muestras malla de 150 m muestreo con laufer de 1,50 y cavado una calicata de $1,75 \mathrm{~m}$. 
Gráfico No1 Mapa Ubicación

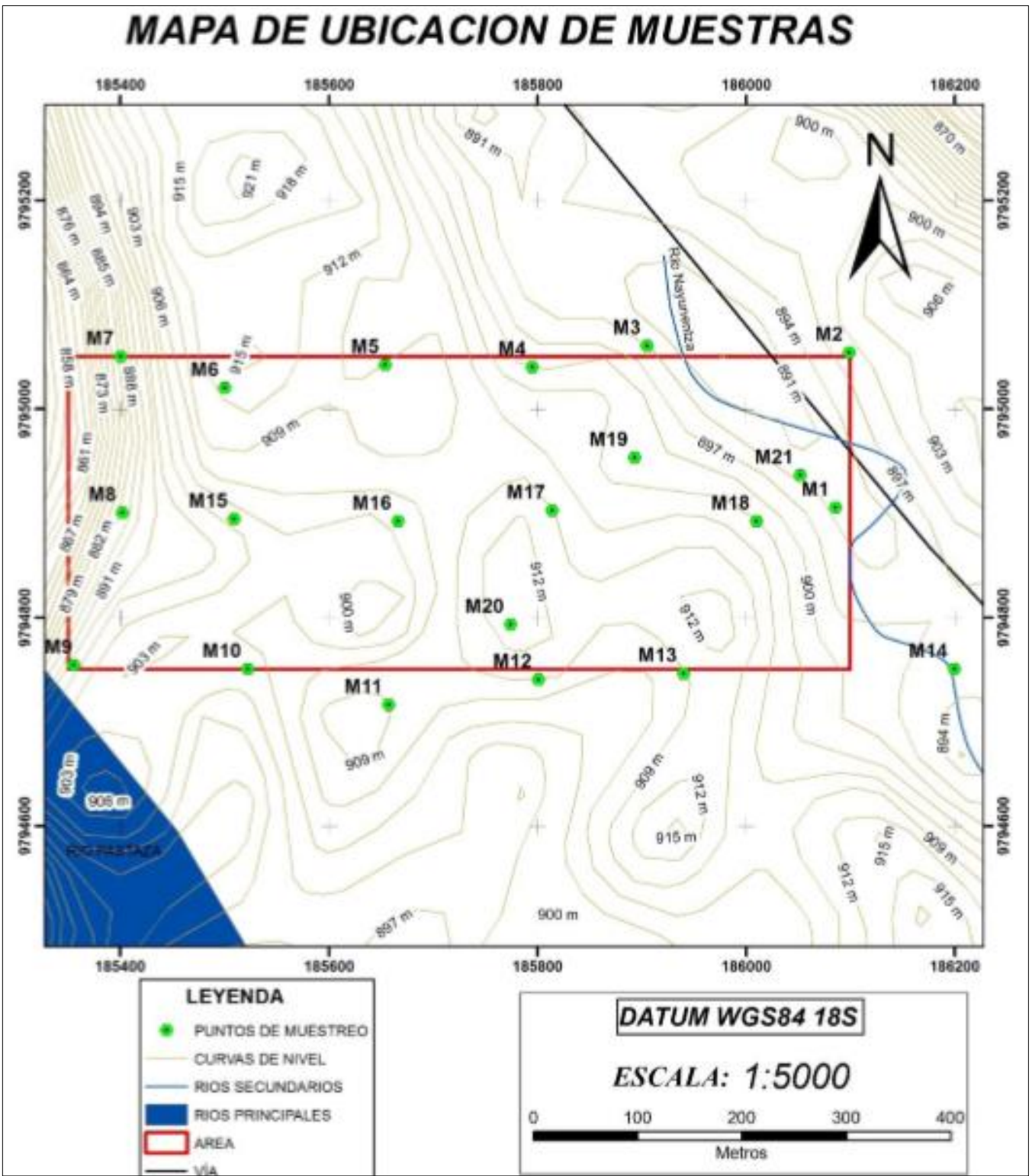

\section{Descripción De Las Zonas De Muestreo}

Básicamente la zona de estudio, se puede observar que por las condiciones geológicas del lugar existe la presencia de un escarpe de aproximadamente 50 metros de altura situada en el borde superior del área de interés.

Litológicamente hablando se puede apreciar que el área es un antiguo afluente por el cual se encuentra una depositación de material sedimentario de tipo aluvial en el fondo aproximadamente a 1 metro de profundidad, más cerca de la superficie se aprecia una capa de arcilla, la cual se encuentra altamente saturada ya que en ciertas partes se forman pantanos lo que dificultó la tarea de muestro. 
Gráfico No2 Descripción de las fichas de muestreo

A continuación se presenta la descripción de las 22 muestras tomada en el área de estudio en las cuales se definen coordenadas Este y Norte, profundidad a la cual fue tomada la muestra, descripción de la muestra

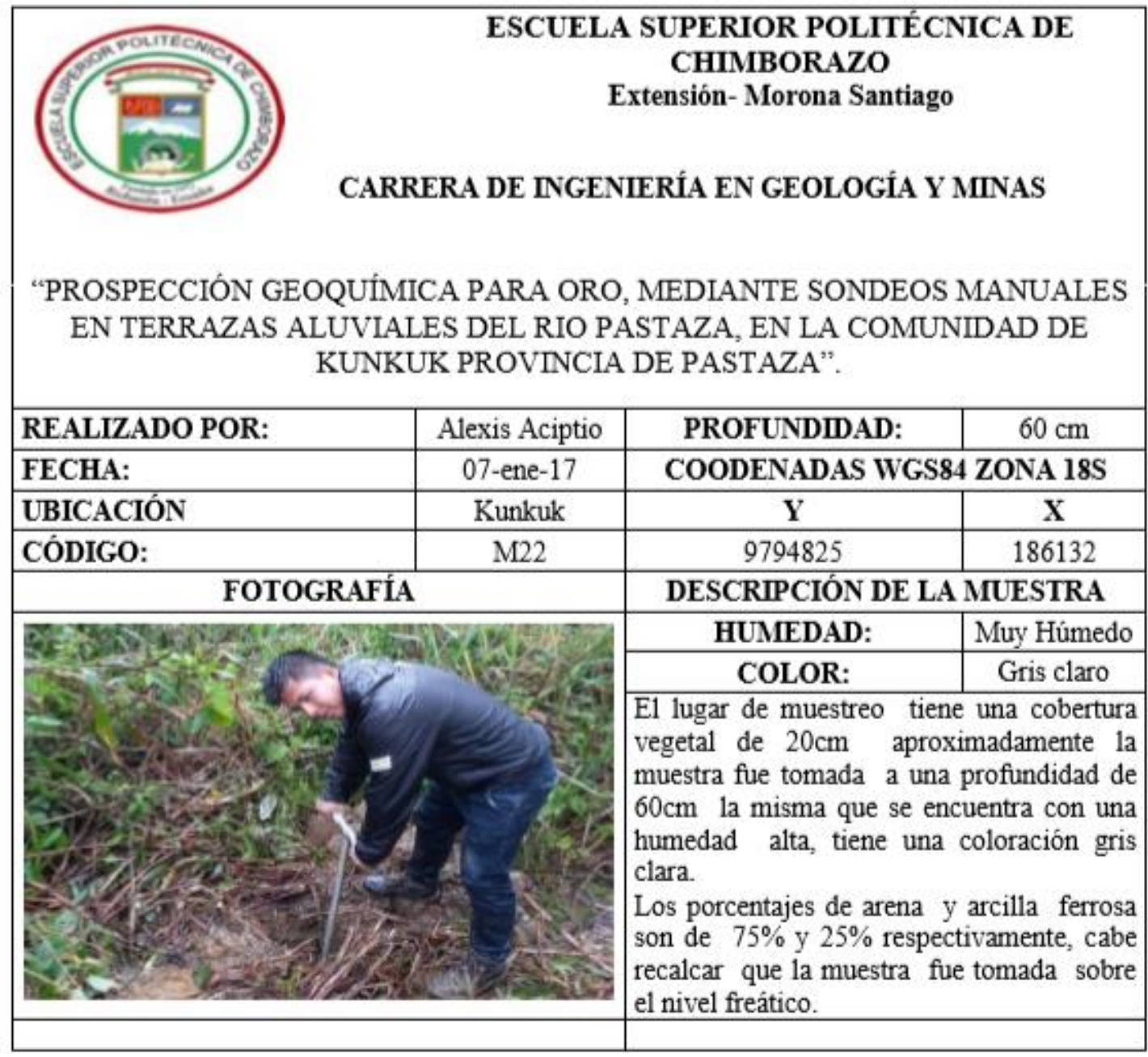

\section{Análisis Geoquímico De Oro}

Las muestras obtenidas con el trabajo de campo, fueron analizadas cuidadosamente en el Laboratorio Metalúrgico ALBEXXUS Cía. Ltda. Para ser analizadas y obtener los valores de Oro total se utilizó la Técnica de Ensayo al Fuego. A continuación de describe a detalle los pasos realizados para la preparación de la muestra y obtención de los datos de resultados de las muestras.

Preparación gravimétrica de las muestras.

Previamente se procedió a preparar las muestras de sedimentos, que consiste en homogenizar la muestra hasta en tamaño adecuado con el cual se pueda realizar el proceso de fundición. 


\section{Ciencia

Para preparar la muestra se realizó el etiquetado y codificado en bandejas de acero inoxidable, evitando que se generen confusiones.

Debido al alto contenido de agua en las arcillas se procedió al secado de las muestras con ayuda de un horno eléctrico a $250^{\circ} \mathrm{C}$, para optimizar tiempos en el desarrollo de la preparación de las muestras se secaron parcialmente con la ayuda de cocinas industriales a temperaturas de $110^{\circ} \mathrm{C}$.

Finalmente, para terminar con el proceso de preparación de la muestra se la procede a triturar con ayuda en nuestro caso de un triturador secundario y el pulverizador para tener un tamaño de 75 micras, pasante tamiz número 200.

\section{Análisis de los Resultados}

Para el análisis e interpretación de los datos obtenidos en laboratorio nos basamos en el método de Lepeltier modificado, mediante el cual definimos los valores anomálicos de un elemento como son el Background es el equivalente a la media aritmética, Threshold igual a la suma de la media aritmética más la desviación estándar, la Sub-anomalía es igual a la suma de dos desviaciones estándar más la media aritmética y la Anomalía definida igual a cuatro desviaciones estándar más la media aritmética, a continuación, se presentan los resultados obtenidos de los cálculos estadísticos realizados.

Tabla No1 Análisis de Concentración del Oro

\begin{tabular}{|cc|}
\hline MUESTRA & CONCENTRACION (Gr/Ton, ppm) \\
\hline M1 & $<0,01$ \\
\hline M2 & 0,31 \\
\hline M3 & 0,07 \\
\hline M4 & $<0,01$ \\
\hline M5 & $<0,01$ \\
\hline M6 & $<0,01$ \\
\hline M7 & $<0,01$ \\
\hline M8 & $<0,01$ \\
\hline M9 & $<0,01$ \\
\hline M10 & $<0,01$ \\
\hline M11 & $<0,01$ \\
\hline M12 & $<0,01$ \\
\hline M13 & $<0,01$ \\
\hline M14 & $<0,01$ \\
\hline M15 & 0,2 \\
\hline M16 & $<0,01$ \\
\hline M17 & $<0,01$ \\
\hline M18 & $<0,01$ \\
\hline M19 & $<0,01$ \\
\hline M20 & $<0,01$ \\
\hline M21 & $<0,01$ \\
\hline M22 & $<0,01$ \\
\hline
\end{tabular}

\section{Análisis Univariable}




\section{Ociencia

\begin{tabular}{|cccc|}
\hline Clases & $f i$ & $f r$ & $f a$ \\
\hline $0,01-0,07$ & 20 & 0,91 & 20 \\
\hline $0,08-0,13$ & 0 & 0,00 & 20 \\
\hline $0,14-0,19$ & 0 & 0,00 & 20 \\
\hline $0,20-0,25$ & 1 & 0,05 & 21 \\
\hline $0,26-0,31$ & 1 & 0,05 & 22 \\
\hline
\end{tabular}

Elaborado por: Aciptio, A. (2017)

\begin{tabular}{cc|}
\hline $\mathrm{X}=\left(\mathrm{fi}{ }^{*} \mathrm{Xi}\right) / \mathrm{n}$ & 0,05955 \\
$\mathrm{dsv}=\mathrm{V}\left(\sum\left(\mathrm{Xi} 2^{*} \mathrm{fi} / \mathrm{n}\right)-(\mathrm{X} 2)\right)$ & 0,06247 \\
\hline
\end{tabular}

\begin{tabular}{|cccccc|}
\hline \multicolumn{6}{|c|}{ VALORES NORMALES Y ANOMÁLICOS DE ORO } \\
\hline $\begin{array}{c}\text { MEDIA } \\
\text { ARIMETICA }\end{array}$ & DESVIACION ESTANDAR & BACKGROUND & THRESHOLD & SUBANOMALÍA & ANOMALÍA \\
\hline $\mathrm{X}=\left(\mathrm{fi}{ }^{*} \mathrm{Xi}\right) / \mathrm{n}$ & $\mathrm{dsv}=\mathrm{V}\left(\sum\left(\mathrm{Xi2}{ }^{*} \mathrm{fi} / \mathrm{n}\right)-(\mathrm{X} 2)\right)$ & $\mathrm{X}$ & $\sigma+\mathrm{X}$ & $2 \sigma+\mathrm{X}$ & $3 \sigma+\mathrm{X}$ \\
\hline $0,0595 \mathrm{ppm}$ & $0,0625 \mathrm{ppm}$ & $0,0595 \mathrm{ppm}$ & $0,1220 \mathrm{ppm}$ & $0,1845 \mathrm{ppm}$ & $0,2469 \mathrm{ppm}$ \\
\hline
\end{tabular}

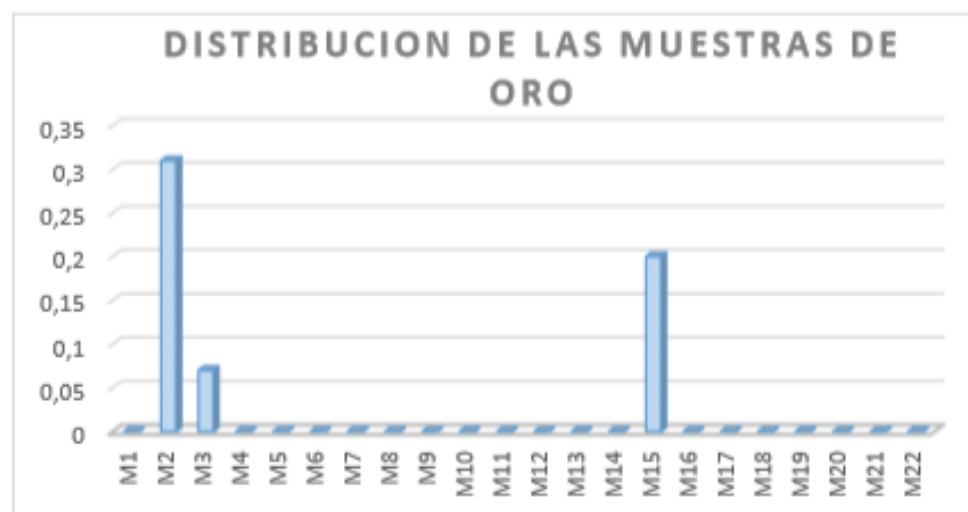

\section{Delimitación de la Zona Anomálica}

Se determinó 2 zonas de concentraciones anomálicas en el área de estudio la primera de menor concentración al oeste del área de estudio y la segunda más importante ubicada al Noreste del área de trabajo con mayor concentración, haciendo un estudio de la distribución del oro en el sector podemos inferir que la depositación del oro secundario viene de la parte alta del sector ubicada al nor-este del área de estudio, también esto se debe a que en la parte con mayor concentración de oro se forma una zona pantanosa lo que implica mayor concentración de sedimentos en dicho sector. La zona anomálica de menor concentración se ubica al borde de un escarpe en la cual se aprecia acumulación de arenas finas con contenido de oro la misma que podemos inferir ha sido producto de una segunda acumulación siguiendo la red de drenaje de la zona pantanosa 
Gráfico No4 Mapa anomalías

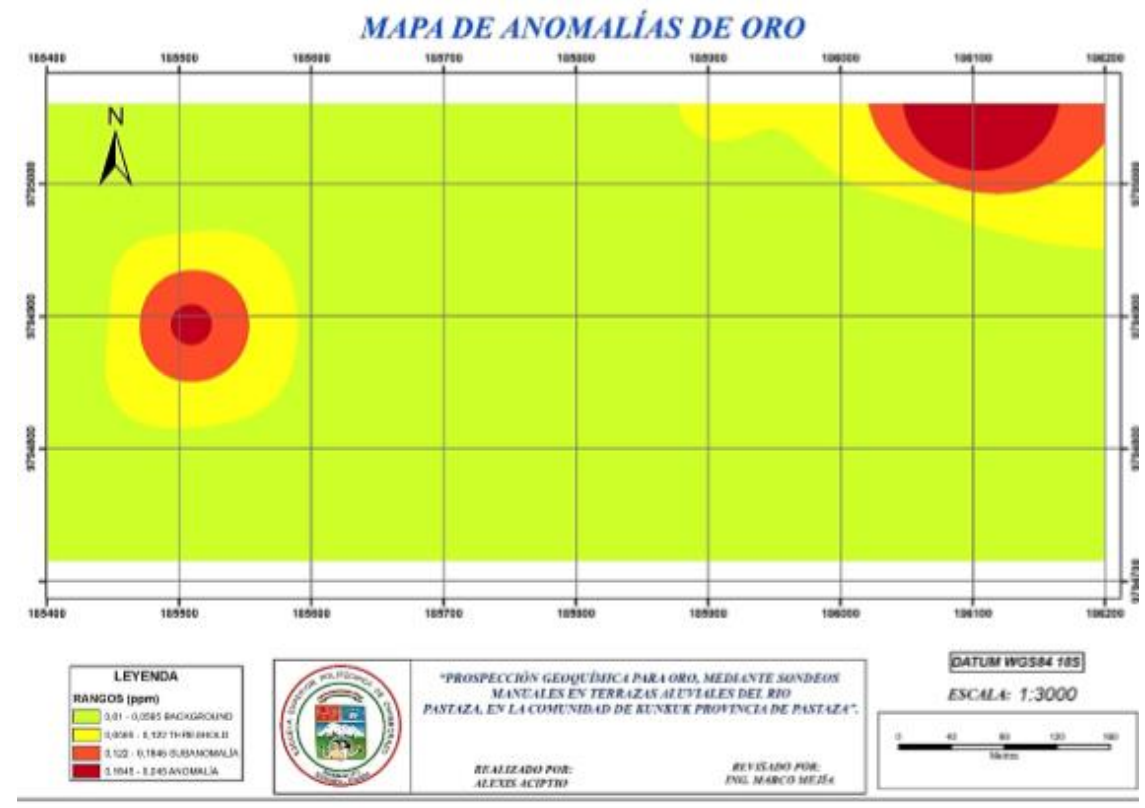

\section{Conclusiones}

- Se determinó una concentración normal de oro para el área de estudio; en el Background 0.595ppm y en el Threshold 0.122 ppm de un análisis de 22 muestras distribuidas uniformemente en una malla con variación en función a la morfología del terreno.

- Mediante el análisis univariable se logró determinar subanomalías de 0.1845 ppm las cuales se concentran en dos puntos; la primera al noreste y la segunda al suroeste.

- Por medio del análisis geoestadístico de Lepeltier modificado se logró determinar las anomalías de $0.2469 \mathrm{ppm}$, en dos sectores con ubicación similar a las subanomalìas.

- El método geoquímico "ensayo al fuego" determinó que en el área de estudio existen zonas de concentración de oro en terrazas aluviales.

\section{Referencias Bibliográficas}

Ayala, V. (2015). Plan de desarrollo y ordenamiento territorial de la Provincia de Pastaza. Baldock. (1982). Formación mera del cuaternario. Recuperado de http://bibdigital.epn.edu.ec/bitstream/15000/2441/1/CD-0142.pdf Foster, (1992). Ensayos geoquímicos, hidrogeología ambiental. Recuperado de http://sedici.unlp.edu.ar/bitstream/handle/10915/15910/Hidrogeolog\%EDa+ambiental+e ntero.pdf;jsessionid=DC2A31478A72A752A277719C11DF958E?sequence=5 GriemKlee. (28 de noviembre de 2016). 
Apuntes geología. Geoquímica en la prospección. Recuperado el 25 de diciembre del 2017, de https://www.geovirtual2.cl/EXPLORAC/TEXT/0300-geoquimicaexploracion.htm 


\section{PARA CITAR EL ARTÍCULO INDEXADO.}

Mejía, M., \& Aciptio, A. (2019). Prospección geoquímica para oro, en terrazas aluviales del rio Pastaza. Ciencia Digital, 3(3.1), 375-389. https://doi.org/10.33262/cienciadigital.v3i3.1.709

El artículo que se publica es de exclusiva responsabilidad de los autores y no necesariamente reflejan el pensamiento de la Revista Explorador Digital.

El articulo queda en propiedad de la revista y, por tanto, su publicación parcial y/o total en otro medio tiene que ser autorizado por el director o editor de la Revista Explorador Digital.

\section{Liencia}

